\title{
INNOVATIVE GROWTH IN DEVELOPING NEW METHODS FOR FORMULATING SOLID LIPID NANOPARTICLES AND MICROPARTICLES
}

\author{
*Kaur Jaspreet ${ }^{1}$, Singh Gurpreet ${ }^{1}$, Saini Seema ${ }^{1}$, Rana AC ${ }^{2}$ \\ ${ }^{1}$ Department of Pharmaceutics, Rayat Institute of Pharmacy, Railmajra, Punjab, India \\ ${ }^{2}$ Department of Pharmacology, Rayat Institute of Pharmacy, Railmajra, Punjab, India \\ Corresponding Author's E-mail: saini.jaspreet08@yahoo.com
}

Received 28 July 2012; Review Completed 11 Sep 2012; Accepted 11 Sep 2012, Available online 15 Sep 2012

\begin{abstract}
The solid lipid nanopaticles are the colloidal drug delivery system which is spherical in shape and present in 10-1000nm particle sized range. The surfactant is used to stabilize the solid lipid nanoparticles to avoid aggregation. The Co-surfactant was used in addition to increase the micelles concentration. The optimization of surfactant concentration was used to stabilize the nanoparticles and microparticles formed and to decrease the particle size by decrease the aggregation. The manufacturing methods of the SLN are of four types, the high pressure homogenization which is further divides into two types hot homogenization method and cold homogenization method, Solvent-Diffusion evaporation method, ultrasonication method, and membrane contactor method. The non-uniform particles sizes were obtained from high pressure and ultra-sonication methods but the solvent-Diffusion Evaporation method and membrane contactor methods give uniform sized particles. But disadvantages of the later methods are the toxicity of organic solvent if not evaporates completely from the SLNs and the high cost of membrane contactor instrument is there in the formulation of SLNs. While the ultra-turrax and high pressure homogenization methods are the safest methods to use as compared to Solvent-Diffusion Evaporation method. The unpublished results have indicated that the drug release from the HPH and ultrasonication gave highest drug release as compared to marketed conventional gel and formulation obtained by Solvent-Diffusion Evaporation method.

Keywords: SLN, high pressure homogenization method, Solvent-Diffusion Evaporation method, ultrasonication method, Membrane contactor method
\end{abstract}

Abbreviations: NM indicates Nano-meter, SLN: Solid lipid nanoparticle, HPH: High Pressure Homogenizer, PEG: PolyethyleneGlycol

\section{INTRODUCTION}

1.1 Controlled drug delivery- The controlled drug delivery systems have been introduced to overwhelm the drawback of fluctuating drug levels associated with conventional dosage forms. The concept of sustained or prolonged release of biologically active agents has been well appreciated and randomized for decades. Basically there are three basic modes of drug delivery, i.e. targeted delivery, controlled release and modulated release. Controlled release refers to the use of a delivery device with the objective of releasing the drug into the patient body at a predetermined rate, or at specific times or with specific release profiles ${ }^{1}$.

1.1.1 Background of controlled drug delivery - lipid nanoemulsions were introduced during the 50's for the purpose of parenteral nutrition where $10-20 \%$ of total amount of fatty vegetable oils (e.g. soy oil) or middle chain triglycerides in emulsion as a lipid content were used and other ingredients were Phospholipids (stabilizer-0.6-1.5\%) and glycerol (osmorality-regulation, 2.25\%) were added. During recent years more lipophilic formulations were commercialized e.g. etomidate (Etomidat-Lipuro ${ }^{\circledR}$ ) and Diazepam (Diazepam-Lipuro ${ }^{\circledR}$ ) ${ }^{2,3}$. In the beginning of 80 's, Speiser and coworkers developed solid lipid nanoparticles (by spray drying) and Nanopellets for peroral administration. The nanopellets developed by speiser were formulated by mixing melted lipids with high shear homogenizer but it was indicated that it was producing high quantity of microparticles as compared to nanoparticles. Then high pressure homogenization was developed, with

this the $500 \mathrm{~nm}$ sized and low microparticles sized particles were characterized. ${ }^{4}$

\subsubsection{Examples of colloidal particles are as follows:}

1.1.2.1 Dendrimers- Dendrimers are branched macromolecules in which each level of branching is classified as a generation. Generally, dendrimeric polymers developed for drug delivery have a multifunctional hydrophobic inner core that is conjugated to outer hydrophilic moieties, such as PEG. Dendrimeric polymers are promising drug delivery materials because they possess narrow polydispersity and can be designed with many combinations of size, shape, and surface chemistry. ${ }^{5}$

1.1.2.2 Liposomes- liposomes are aqueous compartments enclosed in lipid bilayer membranes and consist of layered lattices of alternating bimolecular sheets of lipids such as phosphatidylcholine, carebrosides, cholesterol etc. They may be unilayered or multilayered and are known as unilamellar or multilamellar liposomes. ${ }^{5}$

1.1.2.3 Nanoemulsion- For a nanoemulsion formulation to include stable particles of a desired size, the interactions between the core- and shell- forming materials often require optimization. This can be done by experimentally by selecting compatible components and determining the appropriate mixing ratio. ${ }^{5}$

1.1.2.4 Micelles formed from linear block copolymerLinear, amphiphilic diblock triblock co-polymers have emerged as the materials of choice for use in a wide range of biomedical applications, including fabrication or coating of biomedical devices, drug delivery, and tissue SSN: 2250-1177 CODEN (USA): JDDTAO 
Jaspreet et al

Journal of Drug Delivery \& Therapeutics; 2012, 2(5), 146-150

engineering. Polymeric micelles comprise a hydrophobic core, which can load and store drugs as cargo, and a hydrophilic shell, which surrounds and solubilizes the hydrophobic core and hinders interactions with components of the host mononuclear phagocytic system. ${ }^{5}$

1.1.2.5 Micelles formed from star-shaped block copolymers- Unimolecular micelles are inherently stable to dilution and may be prepared to be monodisperse and of a smaller size than most multimolecular systems. Star shaped block co-polymers are an excellent material for making unimolecular micelles, provided the molecules can be engineered to avoid self aggregation ${ }^{5}$

1.1.2.6 Solid lipid Nanoparticles- Solid lipid nanoparticles are the nano-carriers which remain solid at room temperature and changes into liquid form while melting at increasing temperature. SLN are composed of $0.1 \%(\mathrm{w} / \mathrm{w})$ to $30 \%(\mathrm{w} / \mathrm{w})$ solid lipid dispersed in an

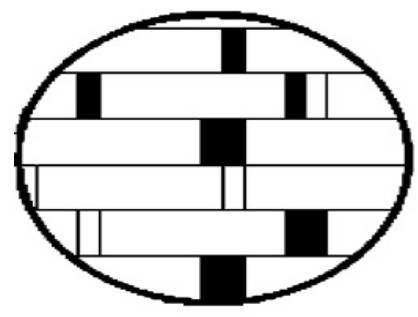

active compound aqueous medium and if necessary stabilized with preferably $0.5 \%(\mathrm{w} / \mathrm{w})$ to $5 \%(\mathrm{w} / \mathrm{w})$ surfactant. The incorporation of cosmetic and pharmaceutical actives is feasible. The mean particle size of SLN is in the submicron rage, ranging from about 40 to $1000 \mathrm{~nm}$. In the second generation of the lipid nanoparticle technology, the particles are produced using blends of solid lipids and liquid lipids (oils). To obtain the blends for the particles matrix, solid lipids are mixed with liquid lipids (oils), preferably in a ratio of 70:30 upto a ratio of 99.9:0.1. The major advantage of SLN is the possibility of production on large industrial scale. However, depending on the drug some potential problems can occur, such as drug leakage during storage and insufficient total drug load. To overcome the limitations of SLN, nanostructured lipid carriers (NLC) have been developed. The later consists of a solid lipid matrix with a high content of liquid lipid. ${ }^{6}$

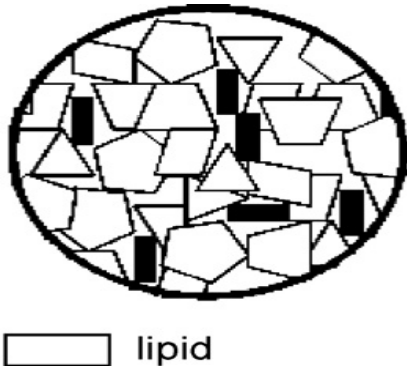

Figure 1.2.1: Formation of an almost perfect crystalline structure in SLN (left) by identically shaped molecules similar to a brick wall with limited loading capacity for actives. Formation of a solid particle matrix of NLC (right) with many imperfections comparable to building a wall from very differently shaped stones, the increased number of imperfections leads to an increased loading capacity for active compounds.

Table 1.2.1: Lipids and emulsifiers used for preparation of solid lipid nanoparticles [4]

\begin{tabular}{|c|c|}
\hline Lipids & Emulsifiers/Coemulsifiers \\
\hline Triglycerides & Soybean lecithin \\
\hline Tricaprin & (LipoidÒ S 75, LipoidÒ S 100) \\
\hline Trilaurin & Egg lecithin (LipoidÒ E 80) \\
\hline Trimyristin & Phosphatidylcholine \\
\hline Tripalmitin & (EpikuronÖ 170, Epikuron 200) \\
\hline Tristearin & Poloxamer 188 \\
\hline Hydrogenated coco-glycerides & Poloxamer 182 \\
\hline (SoftisanÒ) & Poloxamer 407 \\
\hline Hard fat types & Poloxamine 908 \\
\hline WitepsolÒ W 32 & Tyloxapol \\
\hline WitepsolÒ H 35 & Polysorbate 20 \\
\hline WitepsolÒ H 42 & Polysorbate 60 \\
\hline WitepsolÒ E 85 & Polysorbate 80 \\
\hline Glyceryl monostearate (ImwitorÒ900) & Sodium cholate \\
\hline Glyceryl behenate (CompritolÒ 888 ATO) & Sodium glycocholate \\
\hline Glyceryl palmitostearate (PrecirolÒ ATO 5) & Taurocholic acid sodium salt \\
\hline Cetyl palmitate & Taurodeoxycholic acid sodium salt \\
\hline Stearic acid & Butanol \\
\hline Palmitic acid & Butyric acid \\
\hline Decanoic acid & Dioctyl sodium sulfosuccinate \\
\hline Behenic acid & Monooctylphosphoric acid sodium \\
\hline Acidan N12 & \\
\hline
\end{tabular}

In Fig. 1.2.2, the REM of Compritol stabilized with poloxamer 188 is showing the size and shape of the typical solid lipid nanoparticle structure. The table 1.2.1 and 1.2.2 showing the types and properties like melting points, \% of glycerides and hydroxyl value. 


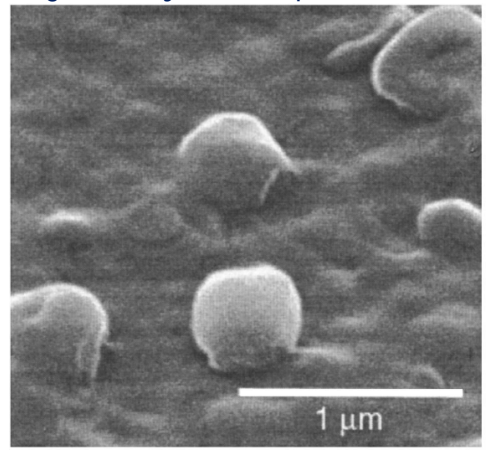

Figure 1.2.2: Picture of solid lipid nanoparticles made from Compritol stabilized with Poloxamer 188, diameter $400 \mathrm{~nm}$.

Table 1.2.2: Chemical composition, melting point and hydroxyl value of lipids used as SLN matrix ${ }^{8}$

\begin{tabular}{|l|l|l|l|l|l|}
\hline Lipid & \multicolumn{2}{l}{ \% of glycerides } & Melting Point & Hydroxyl value \\
\hline & Mono- & Di- & Tri- & $\left.{ }^{\mathbf{0}} \mathbf{C}\right)$ & (mg KOH/g) \\
\hline Dynasan 112 & - & 3 & 96 & $43-47$ & Max. 10 \\
\hline Dynasan 114 & - & 4 & 95 & $55-58$ & Max. 10 \\
\hline Dynasan 116 & - & 3 & 96 & $61-65$ & Max. 10 \\
\hline Dynasan 118 & - & 2 & 97 & $70-73$ & Max. 10 \\
\hline Softisan 138 & - & 11 & 88 & $37-40$ & Max. 15 \\
\hline Softisan 142 & - & 10 & 89 & $42-44$ & Max. 15 \\
\hline Softisan 154 & - & 3 & 96 & $53-58$ & Max. 10 \\
\hline Compritol 888 & $13-18$ & $52-54$ & $28-32$ & $\sim 70$ & $80-105$ \\
\hline Imwitor 900 & $40-50$ & $\sim 40$ & $\sim 5$ & $56-61$ & $300-330$ \\
\hline Precirol Ato 5 & $8 .-17$ & $\sim 54$ & $\sim 30$ & $52-55$ & $90-110$ \\
\hline Witepsol S 51 & 4 & 33 & 62 & $30-32$ & $55-70$ \\
\hline Witepsol S 55 & 10 & 14 & 75 & $33.5-35.5$ & $50-65$ \\
\hline
\end{tabular}

1.1.3The main factors related with SLN administration: 1.2.6.1.1 Method of preparation influences structures of SLN-The drug enriched shell and drug enriched core are of two types of crystallization structures. The former is obtained while repartitioning of the drug while cooling. And the drug enriched core will be found in case the drug precipitates first before the lipid recrystallizes. It is obtained when dissolving a drug (e.g. Prednisolone) in the lipid melt at or close to its saturation solubility. Cooling of the nanoemulsion will lead to a super saturation of drug in the melted lipid and subsequently to the drug crystallization prior to the lipid crystallization.

1.2.6.1.2 Degree of Crystallization- The polymorphic form is also a parameter to determine the drug incorporation. At storage the lipid particles can change from amorphous to crystalline state after recrystallization. Where the main factors to transformation are temperature and water loss in the SLN nanodispersion.

1.2.6.1.3 Adhesiveness- It is a general property of the very fine particles that means nanoparticles of different kinds. Such a lipid film formation will be able to restore a damaged protective lipid film on the skin. Such a film can have occlusive effect.

1.2.6.1.4 Occlusive effect-The occlusive effect of the SLN membrane was evaluated by using Cream base, SLNcontaining cream base and gel formulation were incubate at 24 hours. where TEWL (Transepidermal water loss) was determined for each as the moisture content in the SLN was present. It has shown that, $33 \%$ was moisture present in the skin area as compared to the $23 \%$ of formulation having SLN-Free base. ${ }^{7}$

1.2.6.2

1.2.6.2.1 Advantages of SLN \& NLC-

a. These carriers are composed of physiological and biodegradable lipids of low systemic toxicity and also low cytotoxicity. Most of the used lipids have an approved status or are excipients used in commercially available topical cosmetic or pharmaceutical preparations.

b. The small size of the lipid particles ensures close contact to the stratum corneum and can increase the amount of the drug penetrating into the mucosa or skin.

c. Due to their lipid matrix, controlled release from these carriers is possible.

1.2.6.2.2 Disadvantages of SLN \& NLC-

a. Lipid crystallization modifications- The crystalline state of the molecule will change into amorphous state while storage. So that the drug expulsion could be takes place in the Solid lipid nanoparticles. But it could be overcome by adding liquid chemical penetration enhancer in the solid lipid can enhance the drug loading and prevents the drug expulsion due to defects occurred in the structure of carrier from crystalline to the amorphous state.

b. Difficult to scale up- The probe does not reach to every corner of the container having Emulsion used to reduce particles into nano-sized at the large scale production. 
c. Cost effective-The homogenizer is expensive than instruments used to formulate conventional drug formulations.

d. Particles aggregation could be possible- The aggregation of the particles could be take place because of the nano-sized. ${ }^{7}$

\subsubsection{Methods of Preparation of SLN}

\subsection{High Pressure homogenization}

\section{a. Cold Homogenization method}

\section{b. Hot Homogenization method}

Lipid nanoparticles can be produced by either the hot or cold high pressure homogenization technique. Fig. 1.3 shows schematically the steps of these two methods. The active compound is dissolved or dispersed in melted solid lipid for SLN or in a mixture of liquid lipid (oil) and melted solid lipid for NLC. In the hot homogenization method the lipid melt containing the active compound is dispersed in a hot surfactant solution of the same temperature $\left(5-10{ }^{\circ} \mathrm{C}\right.$ above the melting point of the solid lipid or lipid blend) by high speed stirring. The obtained emulsion (generally called pre-emulsion) is then passed through a high pressure homogenizer adjusted to the same temperature generally applying three cycles at 500 bar or two cycles at 800 bars. In the cold homogenization method, the active containing lipid melt is cooled down. After solidification the mass is crushed and ground to obtain lipid microparticles. The lipid microparticles are then dispersed in a cold surfactant solution yielding a cold pre-suspension of micronized lipid particles. This suspension is passed through a high pressure homogenizer at room temperature applying typically 5-10 cycles at 1500 bar. Addition of SLN or NLC to an existing product, e.g. cream or lotion, is realized by replacing a part of the water phase with concentrated SLN or NLC dispersion. To maintain the lipid content of the original cream or lotion, the lipid content of the original formulation can be reduced about the amount of incorporated lipid from the lipid nanoparticles. The creams and lotions are produced using the established way of production, cooled to about $30{ }^{\circ} \mathrm{C}$, and the concentrated lipid nanoparticles suspension is then admixed applying gentle stirring. Instabilities of lipid nanoparticles in cosmetic or pharmaceutical creams or lotions containing oil droplets that might occur are

aggregation or dissolution. The presence of solid lipid in such formulations can be proven by differential scanning calorimetry (DSC). The particle size can be determined using photon correlation spectroscopy (PCS) or laser diffractometry (LD). Hydrogel formulations (xanthan gum, hydroxyethylcellulose 4000, Carbopol 943 and chitosan) containing SLN or NLC were investigated regarding the physical stability of the lipid nanoparticles. For both lipid nanocarriers a good physical stability was reported. Using high lipid concentrations a final product can be produced in one step. These particle dispersions have a relatively high consistency; they are cream like or almost solid. By PCS, LD and electron microscopy the existence of intact particles can be proven. ${ }^{6}$ In the un-published results of experiment with insoluble drug, the 4 microns sized particles are obtained but was non-uniform sized.

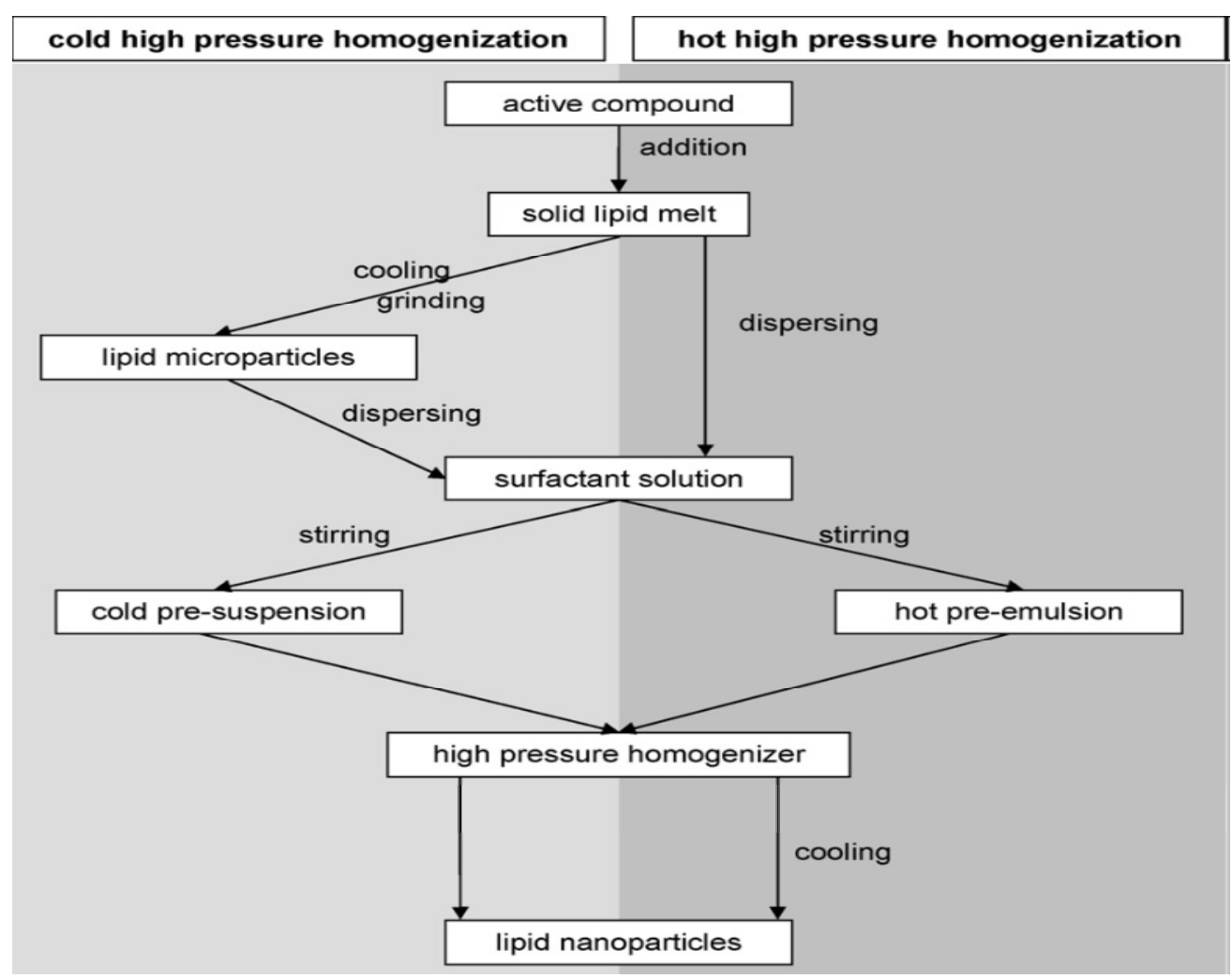

Fig 1.3: Production process of lipid nanoparticles using cold (light gray background) and hot (dark gray background) high pressure homogenization technique ${ }^{6}$

1.2.6.3.2 Solvent-diffusion evaporation method- In this method, the drug and lipid dissolves in organic solvent and put this solution drop wise in the surfactant solution. After stirring, the solution is transferred in the High pressure 
homogenization. The solvent evaporation by Rotaevaporator would be the last step in the SLN Formation. Sjostrom and Bergenstahl described a production method to prepare nanoparticle dispersions by pre-cipitation in $\mathrm{o} / \mathrm{w}$ emulsions. The lipophilic material is dissolved in a waterimmiscible organic solvent (e.g. cyclohexane) that is emulsified in an aqueous phase. Upon evaporation of the solvent nanoparticle dispersion is formed by precipitation of the lipid in the aqueous medium. ${ }^{4}$

1.2.6.3.3 Ultrasonication method- It is also named as high speed homogenization method. High shear homogenization and ultrasound are dispersing techniques which were initially used for the production of solid lipid nanodispersions . Both methods are widespread and easy to handle. However, dispersion quality is often compromised and by the presence of microparticles. Furthermore, metal contamination has to be considered if ultra-sound is used. Ahlin et al. used a Lak Tek rotor-stator homogenizer (Omni International, Gainesville, USA) to produce SLN by melt-emulsification. They investigated the influence of different process parameters, including emulsification time, stirring rate and cooling conditions on the particle size and the zeta potential. Lipids used in this study include trimyristin (DynasanÒ114), tripalmitin Hot (DynasanÒ116), tristearin (DynasanÒ118), a mixture of mono-, di- and triglycerides (WitepsolÒW35, WitepsolÒH35) and glycerol behenate (CompritolÒ888 ATO), poloxamer 188 was used as steric stabilizer (0.5 W\%). ${ }^{4}$

1.2.6.3.4 Membrane contactor- In this method, the lipid phase is pressed, at a temperature above the melting point of the lipid, through the membrane pores allowing the formation of small droplets. The aqueous phase circulates inside the membrane module, and sweeps away the droplets forming at the pore outlets. SLN are formed by the cooling of the preparation at room temperature. The influence of the process parameters (aqueous phase and lipid phase temperatures, aqueous phase cross-flow velocity and lipid phase pressure, membrane pore size) on the SLN sire and on the lipid phase flux was investigated. The 70 and 215 nm sized particles were formulated. ${ }^{9}$

\section{a. Advantages of the membrane contactor method-}

Uniform Sized particles could be obtained as per the pore size.

Mechanical handling of the nanoparticles could be obtained.

No aggregation could take place.

\section{b. Disadvantages of membrane contactor method-}

\section{REFERENCES}

1. Vyas S, Khar RK, Controlled Drug Delivery- Concept and Advances, Vallabh Prakashan, $1^{\text {st }}$ ed., 2002; 418-422.

2. Davis S S, Washington C, West P, illum L, Lipid emulsions as drug delivery systems, Ann N.Y. Acad. Sci 1987; 507:75-88.

3. Graham C W, Pagano R R, Katz R L, Thromboplebitis after intravenous diazepam- can it be prevented, Anesth. Analg.1977; 56: 409-413.

4. Mader K, Mehnert W. Solid lipid nanoparticles Production, Characterization and applications. Advanced drug delivery reviews 2011; 47: 165-196.

5. Huynh L, Neale C, Pomes R, Allen C. Computational approaches to the rational design of nanoemulsions, polymeric micelles, and dendrimers for drug delivery. Nanomedicine: Nanotechnology, Biology, and Medicine 2012; 8: 30-31.
Scalability

Expensive

1.2.6.4 Influences of the Ingredient Composition in the Product Quality of SLN:

1.2.6.4.1 Influence of the emulsifier in SLN Formulation- High concentrations of the emulsifier reduce the surface tension and facilitate the particle partition used during homogenization. The decrease in particle size is connected with a tremendous increase in surface. The increase of the surface area during HPH occurs very rapidly. Therefore, kinetic aspects have to be considered. The process of a primary coverage of the new surfaces competes with the agglomeration of uncovered lipid surfaces. The primary dispersion must contain excessive emulsifier molecules, which should rapidly cover the new surfaces.

1.2.6.4.2 Influence of the lipids concentration in SLN Formulation- Using the hot homogenization, it has been found that the average particle size of SLN dispersions is increasing with higher melting lipids. These are in agreement to the general theory of HPH and can be explained by the higher viscosity of the dispersed phase. However, other critical parameters for nanoparticle formation will be different for the different lipids. Examples include the velocity of lipid crystallization, the lipid hydrophilicity (influence on self-emulsifying properties) and the shape of the lipid crystals (and therefore the surface area). It is also noteworthy, that most of the lipids used represent a mixture of several chemical com pounds. The composition might therefore vary from different suppliers and might even vary for different batches from the same supplier. However, small differences in the lipid composition (e.g. impurities) might have considerable impact on the quality of SLN dispersion (e.g. by changing the zeta potential, retarding crystallization processes etc). By increasing the lipid content over 5-10\% results in larger particles (including micro particles) and broader particle size distributions. Both a decrease of the homogenization efficiency and an increase in particle agglomeration cause this phenomenon which has been observed for lipid nanoemulsions, too. ${ }^{4}$

\section{CONCLUSION:}

The all above formulation methods having many advantages and disadvantages but the high drug release is obtained from the high pressure homogenization of SLN-NLC type formulation. The nonuniformity of the SLNs is there in ultra-sonication method.

6. Muller R H, Pardeike J, Hommoss A. Lipid nanoparticles (SLN, NLC) in cosmetic and Pharmaceutical dermal products. International journal of pharmaceutics 2009; 366: 170-184.

7. Gohla S, Muller R H, Mader K. Solid lipid nanoparticles (SLN) for Controlled drug delivery- a review of the state of the art- Review article. European journal of pharmaceutics and biopharmaceutics 2000; 50: 161-177

8. Soukharev A R, Stability of lipids excipients in solid lipid nanoparticles. Advanced drug delivery reviews 2004; 59: 11-418.

9. Charcosset C, El-Harati A, Fessi H. Preparation of Solid lipid nanoparticles using membrane contactor. Journal of Controlled $\begin{array}{llr}\text { release } & 2005 ; & 108 \text { : }\end{array}$ 